\title{
Fire Detection and Alarm Using Gaussian Blur Background Subtraction Technique
}

\author{
Bhanumathi. M ${ }^{\text {a }}$, Akash Ganesan ${ }^{\text {b }}$, Krishna Kumar. $\mathbf{R}^{\text {b }}$ and Kiruba Wellwin. $\mathbf{J}^{\mathrm{b}}$

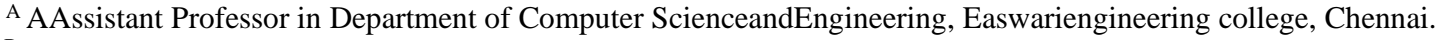 \\ в Undergraduate Student in Department of Computer ScienceandEngineering, Easwariengineering College, Chennai
}

Article History: Received: 11 January 2021; Accepted: 27 February 2021; Published online: 5 April 2021

\begin{abstract}
Fire outbreak is a major issue in oil wells, forests, houses, industries all over the world. The damage caused by these types of accidents is tremendous towards nature and humankind. After these incidents, the need for an application for fire detection has increased. However various fire detection systems use temperature or smoke sensors which takes more response time. Moreover, these systems are expensive and are not widely effective, when the fire is placed far away from the detectors. This led to various alternatives such as computer vision, image processing techniques. One of the cost-effective methods would be the usage of surveillance cameras to detect the fire to inform the relevant parties. The proposed research work suggests a method to use surveillance cameras to monitor the occurrence of fire anywhere within the camera range. In this method, an RGB color pattern and motion detection technologies are used to detect the fire by background subtraction method. The choice capacity of fire-pixels is concluded by the force and immersion of the R part. It has been used that finds the boundary of the moving region in the color segmented image and calculates the number of fire pixels in this area. At that point, a fire identification framework is created dependent on this technique to identify fire effectively to spare life and property from fireperil.
\end{abstract}

Keywords: RGB to grayscale, fore ground mask, GAUSSIAN blur background subtraction, Motion detection.

\section{Introduction}

Fire accident, especially in industries, buildings and in oil wells causes severe damage to many lives of people. The earlier fire detection System is the most significant and efficient method. The mostly practicing methods are installing the burglar alarms in the ceiling. This framework comprises an arrangement of several gadgets cooperating to distinguish and caution individuals through visual and sound apparatuses when fire, smoke are available noticeable all around. These detectors find the fire or smoke by photoelectric and then ionization method. In order the ionize the particle inside the detector it takes more time. Apart from this, they are also a few methods in image processing to detect the fire such as rule-based algorithm, edge detection, etc. In these methods, fire cascade function is used to detect the fire and comparing the different frames continuously. If there are any changes in the frame, then a frame is formed on the screen where there is fire. The model is previously trained with the fire images, now the real-time image is compared with the trained model, and the accuracy rate is displayed on the frame. Subsequently, an alarm is produced. In this method, more noise is produced in the video, so there is more chance for false alarm and detection (Chen, 2004; Toreyin, 2005; Latchoumi, 2020).

The technologies used nowadays are Fire detectors, Smoke detectors, and Temperature detectors. These methods sense the fire not in the beginning state but only when it reaches the severe condition. Further to cover an entire area more detectors have to be installed. It even consumes more money and time to install. The implementation of fire detection using image processing has increased due to the frequent occurrence of fire accidents which results in risking human life and property (Toreyin, 2006; Aroulanandam, 2020). The current fire detection methods are based on electronic sensors that depend on heat, temperature, and pressure sensors. However, these methods have a fatal flaw where they will only work when a certain condition has been reached. If the sensors are damaged or not configured properly, it will cause heavy casualty in case of a real fire (Celik, 2007; Pavan, 2020). To resolve these situations electronics surveillance cameras are installed. There is an increase in the need for fire detection based on image processing and computer vision for such devices. This method is installed in the CCTV camera, UAVS, etc.... There is a growing movement to replace traditional fire detection technologies with computer vision-based solutions, thanks to rapid advancements in digital camera technology and video processing techniques (Raja, 2015; Balamurugan, 2017). Usually, cv-based fire detection systems consist of three stages: fire pixel recognition, moving object segmentation, and candidate area analysis. This procedure is dependent on two factors: the form of the region and the region's time-related shifts. The output of the fire pixel classifier, which creates seed areas for the rest of the device to exercise, is largely responsible for the precision of fire detection. As a result, the fire pixel distinguisher must have a high detection rate and a low false alarm rate. In the literature, only a few algorithms are used for fire pixel classification. Both grayscale and shading video groupings can be used to display the fire pixel designation (Phillips, 2000; Balamurugan, 2020). The vast majority of work on fire pixel classification in shading video successions is focused on rules. Others have established a set of rules to discern the fire pixels using raw R, G, and B color material. Rather than using the rule- 
based color model, which is a combination of Gaussian models in RGB space derived from a training collection of fire pixels, Chen's fire classification system was combined with the motion detection technique of the current methods. Since the tuning parameters used in the background subtraction are so sensitive, the overall method generates a higher rate of false alarms. GMM-based moving foreground segmentation is proposed for HSV color space video sequences in our proposed framework (Balamurugan, 2020). Using GMM-based context deduction, the suggested calculation provides a mask for moving posts. Different learning rates are changed to provide a more precise picture of the context. Inside each moving item separated, cast shadow zones are known. Morphological approaches are used to achieve the integral traveling foreground (Celik, 2006; Chinnamahammad bhasha, 2020; Marbach, 2006)

\section{Literature Review}

A novel based fire flame detection algorithm using color information and motion mechanics. Since fire moves in an irregular direction unlike humans and other objects who moves in a linear direction, it is difficult to fetch the coordinates of the fire'souter region and though we can identify the fire's color using the color information there is a possibility that the algorithm incorrectly identifies an object which has the same color as a fire. This is why the authors have suggested using the motion estimates. The color information of the fire is obtained using an HSI color space and for the motion part, the algorithm uses a combined local-global approach with total variation to determine the various motion vectors of the flame region (Horng, 2005).

Guodong Li et al devised an algorithm to develop a stereoscopic imaging technique for fire detection. The authors experimented with the help of a low-cost CCD camera to obtain the stereoscopic image of the fire. The algorithm computes the disparity of the image pairs and the fire depth is determined by the stereoscopic principle. After calculating the image pairs, image segmentation is done by transforming the RGB color space to HSV color space with which we can quickly identify the information of the fire color. A Scale Invariable Feature Transformation algorithm is used to locate the correspondence points in the stereo images.

\section{Methodology}

\subsection{The Proposed Method}

\subsubsection{CCTV camera}

The proposed system adopts the fire accident according to the real-time image of fire using image processing techniques. This model uses CCTV cameras to sense the changes in the environment due to fire and updates the database. Since CCTV cameras are operational at almost every place these days, it can be used to find a fire at the initial stage and we can take the prevention matters at the beginning.

\subsubsection{Arduino UNO}

Arduino UNO is used to activate the emergency alarm and immediate response to cease the fire.it has14 digital I/O pins.

\subsubsection{WIFI Module}

The ESP8266 WIFI module is used to transmit the signal for response and reflex to cease fire accident.

\section{System architecture}

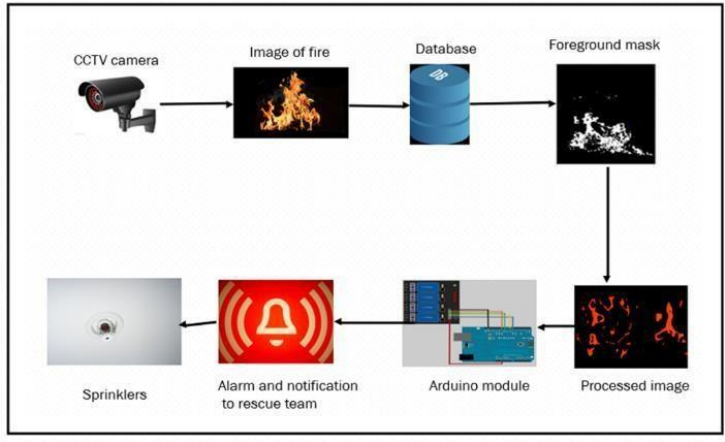

Figure 1. System Architecture

The system that we put forward consists of a CCTV camera that is used to capture the reference image and the actual image. Then actual image of the fire is captured and stored in the database. The captured image is then processed by some image processing techniques like RGB to Gray scale image, background subtraction, 
motion detection, image enhancement . Aftert his all process noises in the image are reduced and fine dimage is obtained. If the value of red in the image is more than a certain value, then the alarm is activated and notification is sent to the rescue team immediately and the sprinklers are activated automatically by using the WIFI Arduino model and a relay is also connected with it, which controls the flow of the water. once the fire accident is cleared it stops the flow of water automatically once the database is updated.

\section{Functional Architecture}

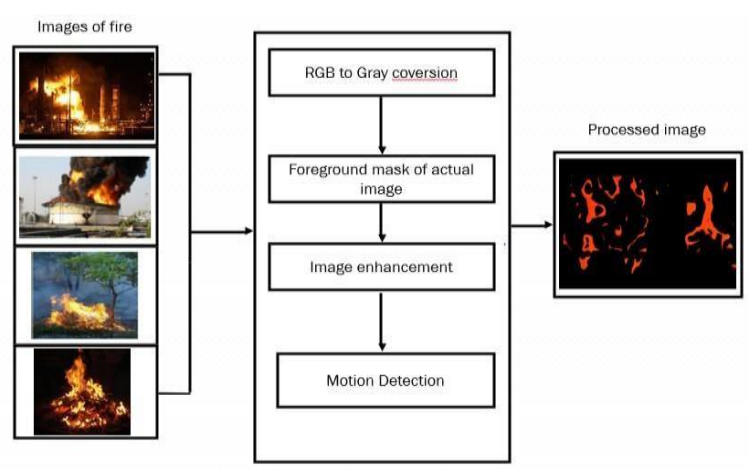

Figure 2. Functional Architecture

The input for the system is different types of images office. After the image is captured then it is converte $\mathrm{d}$ from RGB to Grayscale image. After this Gaussian mixture, the model is done and the fore ground mask of the actual image of fire is obtained. By this method, the noises in the image are reduced and image enhancement is done. Then the motion detection technique is applied in the image, if this condition is accepted then the image is confirmed as fire. As the output, a processed image is obtained which is then compared with the value of the red. If it satisfies the condition then the alarm system is activated

\section{List of Modules}

I. Storing images in the database DB

II. Precaution module

III. Danger state module

IV. Image processing techniques

\subsection{Storing images in the database DB}

In this module images of fire captured by the camera are made to compare with the reference images, which are trained by the model. if the input image is accepted by the system then the image processing techniques are carried out. If the system fails to accept the input image, then it stores the image in the database for further references.

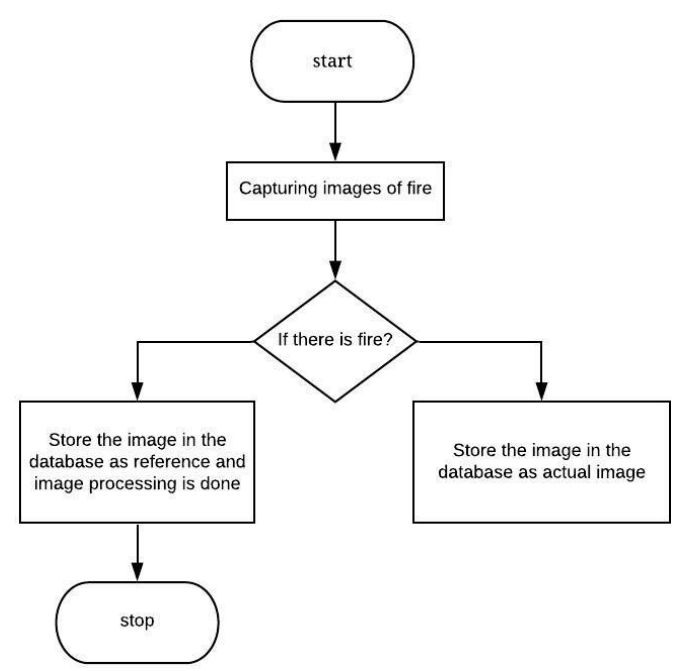

Figure 3. Database module

\subsection{Precaution module}


In this module, Fig 4 illustrates the prevention of fire accidents in an earlier stage. When the fire is detected by the system, then the image processing techniques are done and then the value of red color is calculated from the image. If the value of red in the image is more thanour input value, then the notification to the rescue team and alarm isproduced.

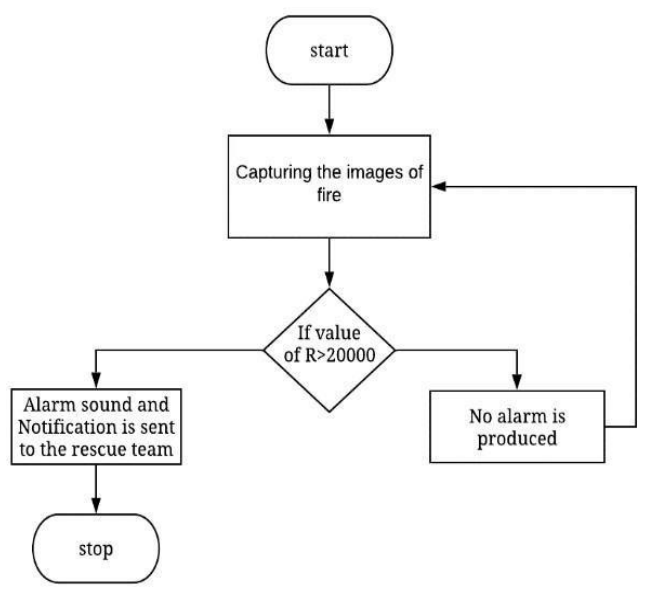

Figure 4. Precaution module

\subsection{Danger State Prevention module}

Fig 5 illustrates the Danger level protocol, the working procedure of the previous model and this are similar and there is an additional feature in this model. If the value of red color from the image is more than 35000 , then the sprinklers are activated automatically by an Arduino board with the relay in it and also alarm and notification are sent to the rescueteam.

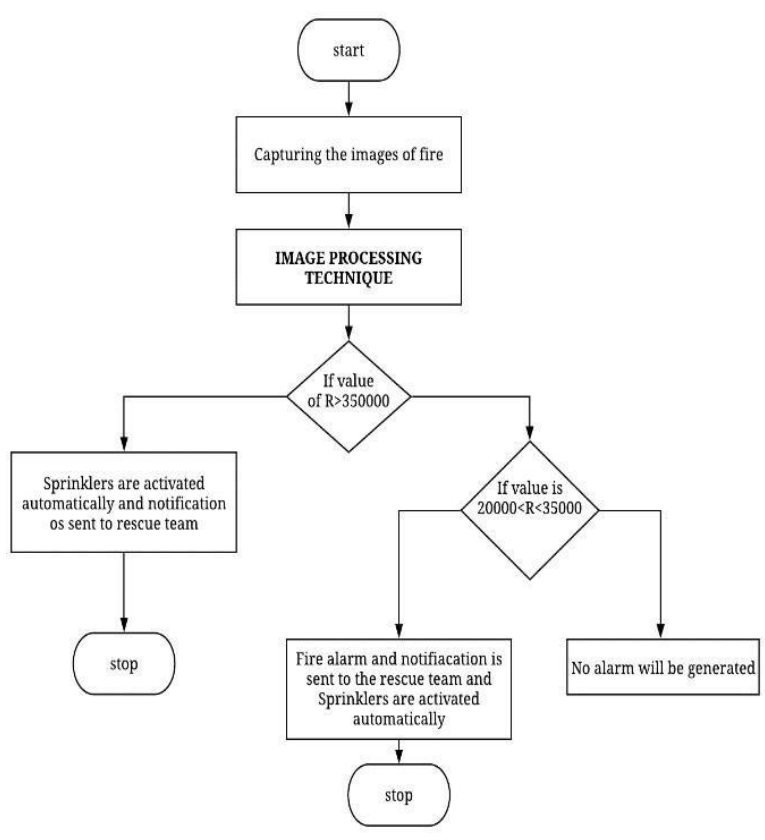

Figure 5. Danger level module

\subsection{Image Processing techniques}

The Fig 6 illustrates the functional procedure of how the image has been processed by the system. In the first stage image of fire is captured and converted into grayscale, to reduce the noise in the image. Then the guassian blur and motion detection is applied to obtain foreground mask of the image. Now value of red color is compared with the mask, Then the alarm is activated according to the previous model. 


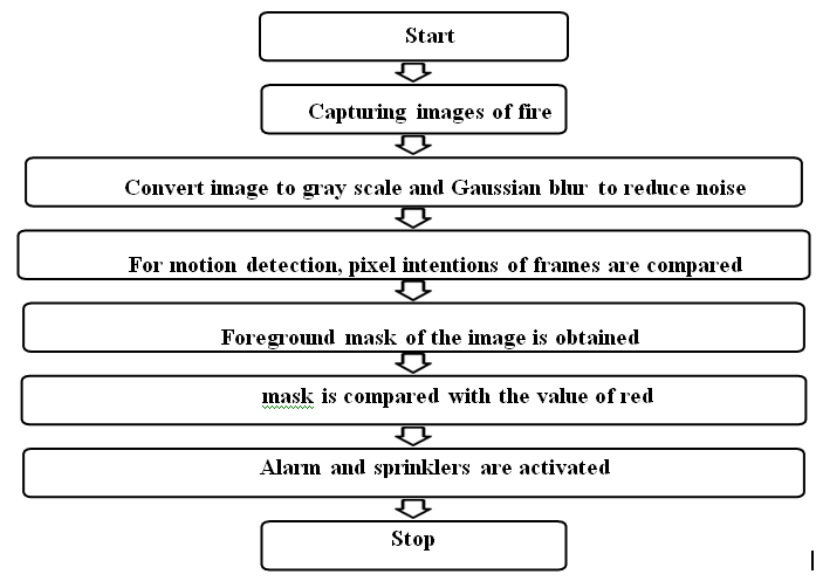

Figure 6. Image processing techniques

\section{Processing chart}

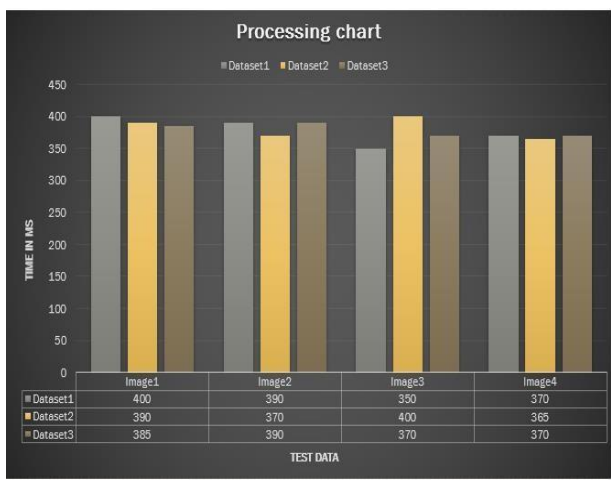

\section{Reference Image of application}

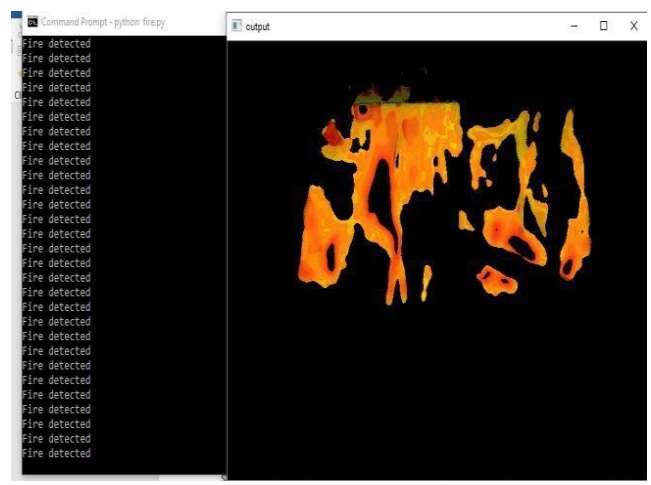

\section{Conclusion}

In this paper, a new image-based continuous fire identification technique was proposed which depends on the computer vision system. The proposed strategy comprises three principal stages, they are fire pixel detection, motion detection, and breaking down fire- shaded moving pixels in back to back frames to raise an alarm. The proposed fire detection model accomplishes a recognition pace of $99 \%$ on the few test videos.

The performance of the proposed system can be advanced my implementing smoke detection at early stages and also even recognizing the very minute amount of fire detection like fire in cigarette, lighter. Even they can cause heavy damage to the industry like an oil well, or some chemical factories, due to negligence of some employees. In some cases, distinguishing smoke is a difficult assignment and inclined to high bogus discoveries caused by mist, diverse lighting conditions brought about ordinarily, and other outside optimal impacts. Sch big bogus identifications can be settled by investigating each smoke-like area. Notwithstanding, this yields a high computational load. The proposed framework accepts that the fire will develop step by step in a spatial area. This 
may not be the situation in certain circumstances. For example, the framework probably won't have the option to identify a fire brought about by an unexpected blast. To mitigate such cases, the proposed framework will be additionally improved to incorporate various situations.

\section{References}

1. Aroulanandam VV, Latchoumi TP, Balamurugan K, Yookesh TL. (2020) Improving the Energy Efficiency in Mobile Ad-Hoc Network Using Learning-Based Routing, Revue d'Intelligence Artificielle, Vol 34(3), pp. 337-343, 2020.

2. Balamurugan K. (2020) Metrological changes in surface profile, chip, and temperature on end milling of M2HSS die steel. International Journal of Machining and Machinability of Materials, 22(6):pp. 443-453.

3. Balamurugan K and Uthayakumar M, (2017) Preparation And Machining Studies Of Lapo4 Y2o3 Ceramic Matrix Composite, http://hdl.handle.net/10603/166221.

4. Balamurugan K, Uthayakumar M, Sankar S, Hareesh US, Warrier KG. (2020) Process optimisation and exhibiting correlation in the exploitable variable of AWJM. International Journal of Materials and Product Technology, 61(1), pp.16-33.

5. Chinnamahammad bhasha A, and Balamurugan, K. (2020) Fracture analysis of fuselage wing joint developed by aerodynamic structural materials." Materials Today: Proceedings, Vol.38, pp. 2555-2562.

6. Chen, T., Wu, P., Chiou, Y. (2004) An Early Fire-Detection Method Based on Image Processing," Proc. IEEE Int. Image Process:1707-1710.

7. Celik. T. et al.( 2007) Fire Detection Using Statistical Color Model in Video Sequences, ” J. Visual Commun. Image Representation,18 (2): 176-185.

8. Celik, T., Demirel, H., Ozkaramanli, H. (2006) Automatic Fire Detection in Video Sequences, ” Proc. European Signal Process. Conf., Florence, Italy.

9. Horng, W.-B., Peng, J.-W., Chen, C.-Y. (2005) A New Image-Based Real-Time Flame Detection Method Using Color Analysis, "Proc. IEEE Networking, Sensing Control :100-105.

10. Latchoumi TP, Reddy MS, Balamurugan K. (2020) Applied Machine Learning Predictive Analytics to SQL Injection Attack Detection and Prevention. European Journal of Molecular \& Clinical Medicine.;7(02), pp. 3543-3553.

11. Marbach, G., Loepfe, M., Brupbacher, T. (2006) An Image Processing Technique for Fire Detectionin Video Images, " Fire Safety J.41(4): 285-289.

12. Raja, K.S., Kiruthika, U. (2015) An Energy Efficient Method for Secure and Reliable Data Transmission in Wireless Body Area Networks Using RelAODV. Wireless Pers Commun83:29752997.

13. Phillips, W., Shah, M. da Vitoria Lobo, N. (2000) Flame Recognition in Video," Proc. 5th Workshop Appl. Computer Vision:224-229.

14. Pavan MV, Balamurugan K, Balamurugan P. (2020) Compressive test Fractured Surface analysis on PLA-Cu composite filament printed at different FDM conditions. InIOP Conference Series: Materials Science and Engineering, Vol. 988,pp. 012019. IOP Publishing.

15. Toreyin, B.U., Dedeoglu, Y., Cetin, A.E. (2005) Flame Detection in Video Using Hidden Markov Models," Proc. IEEE Int. Conf. Image Process., 2005, :1230-1233.

16. Toreyin, B.U., Dedeoglu, Y., Cetin, A.E. (2006) Computer Vision Based Method for Real-Time Fire and Flame Detection," Pattern Recognition Lett. 27( 1) :49-58. 